\title{
Latinx/White Differences in Postsecondary Trajectories: The Role of Parents' Preferences
}

\author{
Amy G. Langenkamp ${ }^{1}$, Nicole Perez ${ }^{2}$ \\ ${ }^{1}$ University of Notre Dame, USA \\ ${ }^{2}$ University of Illinois at Chicago, USA \\ Correspondence: Amy G. Langenkamp, University of Notre Dame, USA.
}

Received: Feb. 12, 2020

doi:10.11114/ijce.v3i1.4750

Accepted: Mar. 1, 2020

Online Published: Mar. 3, 2020

URL: https://doi.org/10.11114/ijce.v3i1.4750

\begin{abstract}
As postsecondary schooling expands, stratification in attainment persists along ethnoracial lines. We build on current research investigating ethnoracial differences in the transition to college by interrogating parents' preference for their child's residence during college. We extend research in two ways. First, we predict whether parents' live-at-home preference is associated with behavior at multiple points in the college-going pipeline. Second, we investigate whether the effect of parents' live-at-home preference differs by ethnoracial group. Results suggest that students whose parents prefer that they live at home are less likely to apply to four-year universities, less likely to attend four-year universities, less likely to enroll full-time among those who are attending four-year universities, and more likely to live with their parents or elsewhere off campus during college. Results also suggest that parents' live-at-home preference has less of an impact on Latinx students' likelihood of applying to and attending four-year universities than white students.
\end{abstract}

Keywords: transition to college, familism, ethnoracial differences

\section{Introduction}

Though access to postsecondary institutions is expanding across ethnoracial groups, inequality persists across these groups in terms of postsecondary destinations (Massey et al., 2003; Posselt et al., 2012) and postsecondary attainment rates (Crisp \& Nora, 2010). For example, Latinx ${ }^{1}$ youth are much more likely to attend two-year colleges (Kurlaender, 2006) and, when attending four-year colleges, have the lowest attainment rates of any ethnoracial group (Krogstad, 2016). Research has tried to make sense of this gap in a variety of ways-investigating preparation and planning for college at early stages of the educational career (Grodsky \& Riegle-Crumb, 2010), ethnoracial group differences net of socioeconomic status and academic achievement in terms of selectivity of postsecondary institution attended (Langenkamp \& Hoyt, 2017a), and the development of informal forms of capital such as college-going social networks (Perez \& McDonough, 2008) and cultural understandings of the admission process (Acevedo Gil, 2017; Alon, 2009; Langenkamp, 2017).

One way to understand differences in postsecondary attainment between Latinx high school students and white high school students has been a focus on differences in familial attitudes towards college. One such behavior that seems to be associated with positive postsecondary outcomes, at least for white families, is the preference to live on campus during college (Desmond \& Lopez Turley, 2009). Parents' preference to have their children live at home during college, or parents' live-at-home preference (LAHP) differs greatly by sociodemographic group and is more common among those who have historically been underrepresented in postsecondary education such as children of immigrants (including Asian and Latinx parents) and those of lower socioeconomic status (Ovink \& Kalogrides, 2015).

The process of status attainment is generally treated as if it would have the same effect on all sociodemographic groups (Bozick et al., 2010); recent evidence is suggesting that ethnoracial minority groups and women do not receive the same benefits simply by mimicking white behavior and attitudes (Langenkamp \& Hoyt, 2017b; Perez \& Ceja, 2015). Therefore, by investigating how an attitude that appears to be advantageous for college-going but that is less prevalent in populations

\footnotetext{
${ }^{1}$ We use this term as an inclusive ethnoracial category for Latina/o individuals living within the current cultural and political context of the United States (Morales, 2018).
} 
of color and immigrant groups might not provide similar benefits for all groups, we can begin to expand upon our models of status attainment and better understand different pathways to college-going.

\section{Literature Review}

\section{The Transition to College}

The transition to college is an important life course transition. The transition to college is also representative of choices and decisions that occur over several years of the educational career (Morgan, 2005). Therefore, the transition to college can be thought of as a process (Carbonaro et al., 2011) with several stages. Parents are important influences on students at each stage in the preparing for college, such as the development of educational aspirations (Ceja, 2004), taking relevant coursework during high school (Morgan, 2005), and applying to college (Perez \& McDonough, 2008).

Research consistently finds that Latinx parents have high educational expectations for their children and are supportive of their children's college-going (Ceja, 2004; Langenkamp, 2017; Valencia, 2002). However, parental support, particularly among parents with lower levels of education, is simply not enough (Klasik, 2012; Langenkamp, 2017). Many Latinx families face obstacles in making high educational expectations a reality in part due to barriers of parental involvement (Alexander et al., 2017), structural barriers of low socioeconomic status (Crosnoe, 2006), where paying for college is a struggle, and segregated schools and neighborhoods (Stanton-Salazar, 2001). While not the focus of the current study, these structural factors act as barriers to the transition to college for many Latinx students (Taggart, 2018).

Parents' level of education also plays a key role in the transition to college. At each stage in the transition to college, students whose own parents went to college are more equipped to navigate the postsecondary admission process and invest in resources to promote college attendance (Alon, 2009). Previous research (Perna, 2000) demonstrates that social inequality is reproduced through the postsecondary choices students make, the resources available to them to help them make those choices, and their experiences during college. Thus, parents with higher levels of education have the "college knowledge" and resources to transmit to their children in their pursuit of entering the college pipeline. Across ethnoracial groups, there is inequality in levels of parental educational attainment; Latinx parents have the lowest average levels of parental education (Crosnoe, 2006). These inequalities in parental educational attainment have implications for various ways that some parents pass on their advantages while others face barriers in providing access to the college pipeline (McDonough, 1997). Because Latinx students are more likely to be first-generation college-going and less likely to have a parent who attended college, many Latinx students are at a disadvantage as they make the transition to college concerning their "college knowledge" (Perez \& McDonough, 2008); college knowledge involves having information about the application process and academic and cultural expectations associated with college-going.

There are certain ways of approaching the transition to college that increase the likelihood of applying to college and enrolling in college, particularly four-year colleges. Where students live during college is an example. Though only $20 \%$ of students live on campus (Perez \& McDonough, 2008), living on campus is associated with retention between the first and second years of college (Schudde, 2011), and may be especially important for students traditionally marginalized on college campuses such as ethnoracial minority students (Fischer, 2007; Turley \& Wodtke, 2010). The positive effects of living on campus are related to students' academic performance and better integration in college social life (Fischer, 2007). However, increased belonging when living on campus is contingent on a positive racial climate at the university (Johnson et al., 2007). Another beneficial action that students take in their transition to college is attending school full-time. Students who attend school full-time during college are more likely to stay in college and to complete their degree (Shapiro et al., 2015). When conceptualizing the transition to college, and even educational attainment, as a process, these interim steps can be viewed as potential opportunities to gain advantages or, alternatively, to lose ground compared to more advantaged actors within a stratified system (Carbonaro et al., 2011; Grodsky, 2007). (Dis)advantage in these interim steps has contributed to stratification along ethnoracial lines in terms of postsecondary attainment, with Latinx students much less likely to graduate from any college, but especially four-year colleges (Shapiro et al., 2015).

\section{Parents'Live-At-Home Preference and Ethnoracial Differences}

One advantage identified by previous research involves familial attitudes. Specifically, research has suggested that a preference to live at home may deter students' application and attendance in college, especially four-year colleges (Desmond \& Lopez Turley, 2009). It is unclear why preference to live at home is detrimental to application and attendance. It may be, as other research has suggested, that parental closeness to college-age children signals an interdependency and, therefore, reluctance on the part of the child to live away from home during college (Lopez Turley et al., 2010). Others have suggested that any reluctance of college-age adolescents to go "away" to college (e.g., live on-campus in college housing) may be mitigated by distance; high school seniors who live closer to a wide-range of college options, including community college and four-year universities, have an increase in the odds that they will apply to four-year college (Desmond \& Lopez Turley, 2009). At the same time, LAHP may simply be a factor of socioeconomic constraint, where living at home is less expensive than living on campus (Ovink \& Kalogrides, 2015). When focusing specifically on 
immigrant families, adolescents may be reluctant to live far away from home both because of the social support and cultural familiarity they find within their family, but also because immigrant children are often important language brokers for their parents when interacting with English speaking institutions such as schools and health care providers (Bean, 2015).

In sum, it is unclear why children and parents report the preference to live at home during college, but empirical findings do consistently suggest that LAHP impedes college-going. Therefore, the preference to live at home during college exists within an important subset of college-going attitudes that students and their families have as they make application and attendance decisions, and, we argue, functions as a mechanism that contributes to inequality. Furthermore, while previous research has focused on application and enrollment stages (Desmond \& Lopez Turley, 2009; Ovink \& Kalogrides, 2015), it is important to also investigate whether a LAHP preference in turn influences some of the college-going behaviors that previous research has identified as beneficial, such as living on campus and full-time enrollment. Incorporating these additional outcomes is a specific contribution of the current study.

Beyond the way that LAHP affects postsecondary outcomes, there are important differences of LAHP by ethnoracial group. Immigrant parents and parents from ethnoracial minority groups are more likely to report a preference for living at home during college (Lopez Turley et al., 2010; Ovink \& Kalogrides, 2015); this in turn is used to partially explain why Latinx students are concentrated in two-year postsecondary institutions. Although normative among white families from higher socioeconomic backgrounds, parents' LAHP is among growing examples that illustrate how the transition to college is not approached in a uniform way. Furthermore, we should not expect that all students have the same experiences when they make the transition to college. For example, ethnoracial minority students have an increased risk of experiences of racism and racial profiling while in college (Yosso, 2006). These students are also more likely to be at risk of social isolation after they transition to college, particularly on four-year college campuses (Fischer, 2007; Ovink \& Veazey, 2011).

\section{Current Study}

The current study builds upon and extends research in two important ways. First, we investigate multiple outcomes in the postsecondary pipeline whereas other studies are limited to one or two outcomes. We initially explore whether parents' LAHP affects college-going behaviors in the application and attendance stages. We also investigate whether parents' LAHP influences important college-going behavior such as full-time versus part-time enrollment and residential living location once students enter postsecondary schooling. The current study provides a way to better understand how one qualitative difference that aligns with both socioeconomic status and ethnoracial differences may affect students' collegegoing pipeline.

Our second extension of research is to use what we know about parents' LAHP to better understand the ways that smaller decisions and attitudes held about college may affect outcomes along the college-going pipeline through our modeling of interaction effects. We consider whether cultural knowledge about normative behavior and attitudes that benefit those who are advantaged (Bourdieu \& Passeron, 1977), in this case living on campus during four-year college, holds the same benefits for Latinx families who espouse similar attitudes and behavior. Although we expect that parents' LAHP of "not important" will positively affect postsecondary pipeline outcomes due to previous research (Desmond \& Lopez Turley, 2009; Ovink \& Kalogrides, 2015), we also expect that the benefit of parents' LAHP will not necessarily extend to Latinx youth's parents who espouse the same LAHP. By investigating the association between parents' LAHP and various outcomes along the college-going pipeline, and how the effect of life-at-home preference may differ between Latinx and white students, we better understand the divergent pathways students are taking when making their transition to college. This is a specific contribution to current research because our approach can help determine whether parents' LAHP operates similarly for Latinx and white youth, or whether there are differential returns to the advantageous preference of living away from home during postsecondary schooling.

\section{Data and Methods}

Research Sample

This study uses data from the Educational Longitudinal Study of 2002 (ELS:2002), a nationally representative longitudinal study in the United States initiated with a cohort of high school sophomores in 2002. In the first year of the study, survey data were also collected from parents, teachers, and school administrators, and the study is further supplemented with high school and postsecondary transcripts. We limited our sample to the following students: nonLatinx white (hereafter, white) and Latinx students who have completed the second follow-up survey in 2006 and had 
either graduated from high school or completed a GED by the time of the second follow-up. ${ }^{2}$ We also restrict the sample to students who have enrolled in postsecondary education after high school $(n=8,686)$. When modeling students' behaviors during college (e.g., attendance full-time, residence during college), we further restrict our sample to students who are currently attending postsecondary schooling $(\mathrm{n}=7,377)$; these data were collected in 2010.

Our data do contain missing on some of the measures; we lose approximately 2,600 cases when deleting missing values $\left(\mathrm{n}=2,100\right.$ white students and $\mathrm{n}=500$ Latinx students). ${ }^{3}$ Multiple imputation was performed using chained multiple imputation $(M=10)$ in order to impute values for missing data among the independent variables. The imputation employed the base-year to third follow-up probability weights in its estimation. Importantly, analyses using listwise deletion produces similar findings to the ones presented here using the multiply imputed sample.

\section{Analytic Plan}

Although previous research has found that parents' LAHP start is associated with both application to college and college attendance, research has done little to better understand why parents hold these preferences. In an effort to unpack what parents' LAHP live-at-home means, we conducted extensive supplemental analyses with the ELS:2002 survey that analyzed whether attitudes, behaviors, and circumstances that may be more prevalent for parents who prefer that their child live at home during college, as well as whether there were any differences between Latinx families and white families. These analyses are discussed in our results section in an effort to help paint a more accurate picture of why parents might have live-at-home preferences, as well as whether these measures explain associations between LAHP and our college-going outcomes.

We use multinomial logistic regression and logistic regression to predict our outcome variables. Models first include ethnoracial group status (reference $=$ white students), adding all other demographic controls in the second model. This establishes associations between ethnoracial group and our outcomes net of other demographic characteristics. Next, in Model 3, we add parents' LAHP. Model 4 include controls for students' high school experiences, such as school contextual factors and academic performance in high school. Finally, in Model 5, we investigate whether the parents' LAHP has a similar effect on Latinx students and white students by modeling interactions between parents' LAHP and ethnoracial group status. Analyses are weighted and account for clustering in schools; because analyses did not include cross-level interactions, multi-level modeling is not necessary.

\section{Results}

\section{Descriptive Statistics}

Table 1 shows descriptive statistics for each of our outcomes and parents' LAHP for white students and Latinx students. Overall, $65 \%$ of our sample has applied to a four-year university and $50 \%$ of these students enroll in a four-year university, with the majority of students' enrolling full time (87\%). This is higher than NCES estimates, likely because NCES measures full-time as year-round enrollment, while our data uses "school year" full time enrollment. Around half of the students in our sample report living on-campus, with $36 \%$ living with parents and very few students (12\%) living offcampus.

\footnotetext{
2 Although our findings did not change when controlling on ethnic differences within the Latinx population, ELS:2002 oversampled from California and Texas, which means that the Latinx sample is mostly Mexican-American. Future research would do well to consider ethnic variation (Nuñez et al., 2008)

${ }^{3}$ The proportion of white and Latinx parents' LAHP is similar even after deleting missing values. Analyses using listwise deletion available from the first author upon request.
} 
Table 1. Descriptive Statistics of Parental Preference and Outcome Variable, by Ethnoracial Group

\begin{tabular}{|c|c|c|c|}
\hline \multirow[b]{2}{*}{ Latinx } & Full Sample & \multirow[t]{2}{*}{ White } & \multirow[t]{2}{*}{ Latino/a } \\
\hline & .18 & & \\
\hline Female & .52 & .52 & .52 \\
\hline \multicolumn{4}{|l|}{ Parents' level of edu } \\
\hline Less than high school & .23 & .19 & .39 \\
\hline Some college or Associate's Degree & .33 & .32 & .34 \\
\hline Four-year degree or higher & .45 & .49 & .27 \\
\hline \multicolumn{4}{|l|}{ Immigrant generation } \\
\hline First generation & .61 & .22 & .25 \\
\hline Second generation & .12 & .53 & .42 \\
\hline Third + generation & .82 & .92 & .33 \\
\hline \multicolumn{4}{|l|}{ Parent's income } \\
\hline Less than $\$ 25 \mathrm{k}$ & .15 & .11 & .32 \\
\hline$\$ 25 \mathrm{k}-50 \mathrm{k}$ & .29 & .27 & .36 \\
\hline$\$ 51 \mathrm{k}-100 \mathrm{k}$ & .39 & .42 & .24 \\
\hline$\$ 101 \mathrm{k}$ or more & .18 & .20 & .08 \\
\hline Single parent household & .18 & .16 & .22 \\
\hline \multicolumn{4}{|l|}{ Parents' live-at-home preference } \\
\hline Not/somewhat important & .75 & .82 & .40 \\
\hline Very important & .25 & .18 & .60 \\
\hline \multicolumn{4}{|l|}{ Urbanicity } \\
\hline Urban & .29 & .25 & .47 \\
\hline Suburban & .50 & .51 & .44 \\
\hline Rural & .21 & .24 & .09 \\
\hline \multicolumn{4}{|l|}{ School sector } \\
\hline Public & .73 & .71 & .83 \\
\hline Catholic & .16 & .17 & .13 \\
\hline Other private & .11 & .12 & .04 \\
\hline High school minority percent & .22 & .15 & .54 \\
\hline High School Grade Point Average (GPA) & 2.84 & 2.92 & 2.49 \\
\hline \multicolumn{4}{|l|}{ Dependent Variables } \\
\hline \multicolumn{4}{|l|}{ Highest level of college application } \\
\hline None & .15 & .14 & .20 \\
\hline Two-Year & .20 & .18 & .28 \\
\hline Four-Year & .65 & .68 & .52 \\
\hline \multicolumn{4}{|l|}{ Level of college attendance } \\
\hline None & .20 & .18 & .29 \\
\hline Two-Year & .30 & .28 & .39 \\
\hline Four-Year & .50 & .55 & .32 \\
\hline Part-time enrollment & .13 & .11 & .24 \\
\hline Full-time enrollment & .87 & .89 & .76 \\
\hline \multicolumn{4}{|l|}{ Residence during college } \\
\hline On-campus & .48 & .52 & .25 \\
\hline With parents & .36 & .63 & .41 \\
\hline Off-campus & .12 & .12 & .12 \\
\hline $\mathbf{N}$ & 8,686 & 7,115 & 1,571 \\
\hline
\end{tabular}

Source: Educational Longitudinal Study of 2002 (ELS:2002) Second Follow-Up Survey (NCES)

In addition, there are some important differences between Latinx students and white students in our outcomes. Fewer Latinx students apply to four-year universities compared to white students, and more Latinx students do not apply to any postsecondary institution. This pattern is exacerbated when looking at four-year attendance, with only $32 \%$ of Latinx high school graduates attending a four-year university while over half of white students do so. Students attending four-year universities generally have high rates of full-time enrollment, though here, too, Latinx students have the lowest rate of 
full-time enrollment at $76 \%$ compared to a sample average of $87 \%$. Among those who attend four-year postsecondary institutions, white students live on-campus at higher rates. Over half of white students live on campus, compared to $25 \%$ of Latinx students. In supplemental analyses, analyses suggest that economic factors are associated with students' choice of whether or not to live on-campus. College students whose parents have an advanced degree or bachelor's degree live on campus at rates of $83 \%$ and $74 \%$ respectively. Students' whose parents have some college (including an associate's degree) or less than high school levels of education live on-campus at rates of $63 \%$ and $54 \%$ respectively.

Table 1 also illustrates differences in parents' LAHP. Consistent with previous research (Ovink \& Kalogrides, 2015), 60\% of Latinx parents in our sample report "very important" for their preference that their child lives at home during college, compared to only $18 \%$ of white students. We now turn to our multivariate analyses, which predict various outcomes along the postsecondary pipeline.

\section{College Application and Attendance}

Table 2a shows selected coefficients from models predicting college application. When predicting two-year application, Latinx youth are not different from white youth in their likelihood of applying to two-year college. In addition, parents' strong LAHP does not significantly predict two-year application. On the other hand, we see in Table 2a that Latinx students are less likely to apply to four-year colleges compared to white students. Interestingly, this is not the case after controlling for high school achievement (see Model 4). However, that Latinx students are statistically different from white students in our final model, which includes interactions between ethnoracial group and parents' LAHP. Finally, Table 2a shows that, consistent with previous research, parents' level of education and immigrant generation $\left(1^{\text {st }}\right.$ generation compared to $3^{\text {rd }}$ generation) predict students' likelihood of applying to both two-year and four-year college. 
Table 2a. Multinomial logistic regression predicting student's college application: reference category (none)

\begin{tabular}{|c|c|c|c|c|c|c|c|c|c|c|}
\hline \multicolumn{11}{|l|}{ Outcome: Two-Year } \\
\hline & Model 1 & & Model 2 & & Model 3 & & Model 4 & & Model 5 & \\
\hline Latinx student & $\begin{array}{l}1.17 \\
(.11)\end{array}$ & & $\begin{array}{l}1.04 \\
(.15)\end{array}$ & & $\begin{array}{r}.95 \\
(.15)\end{array}$ & & $\begin{array}{r}1.06 \\
(.20)\end{array}$ & & $\begin{array}{r}.81 \\
(.18)\end{array}$ & \\
\hline \multicolumn{11}{|l|}{ Parents' level of edu (ref. = less than $H S)$} \\
\hline Some college or Associate's Degree & & & $\begin{array}{l}1.67 \\
(.17)\end{array}$ & $* * *$ & $\begin{array}{l}1.55 \\
(.18)\end{array}$ & $* * *$ & $\begin{array}{l}1.58 \\
(.19)\end{array}$ & $* * *$ & $\begin{array}{l}1.57 \\
(.18)\end{array}$ & $* * *$ \\
\hline Four-year degree or higher & & & $\begin{array}{l}1.51 \\
(.19)\end{array}$ & $* * *$ & $\begin{array}{l}1.54 \\
(.23)\end{array}$ & $* *$ & $\begin{array}{l}1.46 \\
(.22)\end{array}$ & $*$ & $\begin{array}{l}1.44 \\
(.21)\end{array}$ & $*$ \\
\hline \multicolumn{11}{|l|}{ Immigrant generation (ref. $=$ first generation) } \\
\hline Second generation & & & $\begin{array}{l}1.11 \\
(.22)\end{array}$ & & $\begin{array}{l}1.01 \\
(.23)\end{array}$ & & $\begin{array}{l}1.05 \\
(.24)\end{array}$ & & $\begin{array}{l}1.09 \\
(.25)\end{array}$ & \\
\hline Third + generation & & & $\begin{array}{l}0.67 \\
(.13)\end{array}$ & $*$ & $\begin{array}{r}.54 \\
(.12)\end{array}$ & $* *$ & $\begin{array}{r}.55 \\
(.13)\end{array}$ & $*$ & $\begin{array}{r}.60 \\
(.14)\end{array}$ & $*$ \\
\hline \multicolumn{11}{|l|}{ Parents' LAHP (ref. = not/somewhat important) } \\
\hline Very important & & & & & $\begin{array}{r}.89 \\
(.10)\end{array}$ & & $\begin{array}{r}.94 \\
(.11)\end{array}$ & & $\begin{array}{r}.85 \\
(.10)\end{array}$ & \\
\hline Grade Point Average (GPA) & & & & & & & $\begin{array}{l}1.51 \\
(.11)\end{array}$ & $* * *$ & $\begin{array}{l}1.52 \\
(.11)\end{array}$ & $* * *$ \\
\hline \multicolumn{11}{|l|}{ Interactions } \\
\hline Latinx student * LAHP very important & & & & & & & & & $\begin{array}{l}1.66 \\
(.41)\end{array}$ & $*$ \\
\hline Constant & $\begin{array}{l}1.25 \\
(.06)\end{array}$ & $* * *$ & & & $\begin{array}{l}1.19 \\
(.31)\end{array}$ & & $\begin{array}{r}.30 \\
(.11)\end{array}$ & $* *$ & $\begin{array}{r}.29 \\
(.10)\end{array}$ & $* * *$ \\
\hline \multicolumn{11}{|l|}{ Outcome: Four-Year } \\
\hline & Model 1 & & Model 2 & & Model 3 & & Model 4 & & Model 5 & \\
\hline Latinx student & $\begin{array}{r}.56 \\
(.05)\end{array}$ & $* * *$ & $\begin{array}{r}.71 \\
(.10)\end{array}$ & $*$ & $\begin{array}{r}.69 \\
(.11)\end{array}$ & $*$ & $\begin{array}{r}.95 \\
(.18)\end{array}$ & & $\begin{array}{r}.61 \\
(.14)\end{array}$ & $*$ \\
\hline \multicolumn{11}{|l|}{ Parents' level of edu (ref. = less than HS) } \\
\hline Some college or Associate's Degree & & & $\begin{array}{l}1.92 \\
(.18)\end{array}$ & $* * *$ & $\begin{array}{l}1.58 \\
(.17)\end{array}$ & $* * *$ & $\begin{array}{l}1.63 \\
(.20)\end{array}$ & $* * *$ & $\begin{array}{l}1.61 \\
(.20)\end{array}$ & $* * *$ \\
\hline Four-year degree or higher & & & $\begin{array}{l}3.83 \\
(.41)\end{array}$ & $* * *$ & $\begin{array}{l}3.23 \\
(.40)\end{array}$ & $* * *$ & $\begin{array}{l}2.37 \\
(.33)\end{array}$ & $* * *$ & $\begin{array}{l}2.32 \\
(.33)\end{array}$ & $* * *$ \\
\hline \multicolumn{11}{|l|}{ Immigrant generation (ref. = first generation) } \\
\hline Second generation & & & $\begin{array}{l}1.12 \\
(.22)\end{array}$ & & $\begin{array}{l}1.02 \\
(.22)\end{array}$ & & $\begin{array}{l}1.30 \\
(.32)\end{array}$ & & $\begin{array}{l}1.37 \\
(.34)\end{array}$ & \\
\hline Third + generation & & & $\begin{array}{r}.65 \\
(.12)\end{array}$ & * & $\begin{array}{r}.48 \\
(.10)\end{array}$ & $* *$ & $\begin{array}{r}.56 \\
(.14)\end{array}$ & $*$ & $\begin{array}{r}.63 \\
.16)\end{array}$ & \\
\hline \multicolumn{11}{|l|}{ Parents' LAHP (ref. $=$ not/somewhat important $)$} \\
\hline Very important & & & & & $\begin{array}{r}.49 \\
(.05)\end{array}$ & $* * *$ & $\begin{array}{r}.62 \\
(.07)\end{array}$ & $* * *$ & $\begin{array}{r}.52 \\
(.06)\end{array}$ & $* * *$ \\
\hline Grade Point Average (GPA) & & & & & & & $\begin{array}{l}5.58 \\
(.43)\end{array}$ & $* * *$ & $\begin{array}{l}5.58 \\
(.43)\end{array}$ & $* * *$ \\
\hline \multicolumn{11}{|l|}{ Interactions } \\
\hline Latinx student * LAHP very important & & & & & & & & & $\begin{array}{l}2.40 \\
(.63)\end{array}$ & $* *$ \\
\hline Constant & $\begin{array}{l}3.88 \\
(.16)\end{array}$ & $* * *$ & $\begin{array}{r}.91 \\
(.19)\end{array}$ & & $\begin{array}{l}2.11 \\
(.51)\end{array}$ & $* *$ & $\begin{array}{r}.02 \\
(.01)\end{array}$ & $* * *$ & $\begin{array}{r}.02 \\
.01)\end{array}$ & $* * *$ \\
\hline BIC & 10372 & & 9788 & & 9702 & & 8558 & & 8571 & \\
\hline
\end{tabular}

Model 2 includes added demographic controls; Model 4 includes school context measures

Coefficients for results are reported as Relative Risk Ratios (RRR). Robust standard errors reported in parentheses $\mathrm{p}<0.001,{ }^{* *} \mathrm{p}<0.01,{ }^{*} \mathrm{p}<0.05$ 
Table 2b. Multinomial logistic regression predicting student's college attendance: reference category (none)

\begin{tabular}{|c|c|c|c|c|c|c|c|c|c|c|}
\hline Outcom & & & & & & & & & & \\
\hline & Model 1 & & Model 2 & & Model 3 & & Model 4 & & Model 5 & \\
\hline \multirow[t]{2}{*}{ Latina/o } & 0.86 & $*$ & .89 & & .92 & & 1.13 & & .86 & \\
\hline & $(.06)$ & & $(.09)$ & & $(.10)$ & & $(.15)$ & & $(.14)$ & \\
\hline \multicolumn{11}{|l|}{ Parents' level of edu (ref. = less than HS) } \\
\hline \multirow[t]{2}{*}{ Some college or Associate's Degree } & & & 1.63 & $* * *$ & 1.47 & $* * *$ & 1.50 & $* * *$ & 1.49 & $* * *$ \\
\hline & & & (.13) & & $(.13)$ & & (.14) & & $(.14)$ & \\
\hline \multirow[t]{2}{*}{ Four-year degree or higher } & & & 2.24 & $* * *$ & 2.16 & $* * *$ & 1.94 & $* * *$ & 1.91 & $* * *$ \\
\hline & & & $(.21)$ & & $(.23)$ & & $(.22)$ & & $(.22)$ & \\
\hline \multicolumn{11}{|c|}{ Immigrant generation (ref. = first generation) } \\
\hline \multirow[t]{2}{*}{ Second generation } & & & .98 & & .94 & & 1.00 & & 1.04 & \\
\hline & & & $(.15)$ & & $(.16)$ & & $(.17)$ & & $(.18)$ & \\
\hline \multirow[t]{2}{*}{ Third + generation } & & & .65 & $* *$ & .60 & $* *$ & .63 & $*$ & .68 & * \\
\hline & & & $(.10)$ & & $(.10)$ & & $(.11)$ & & $(.12)$ & \\
\hline \multicolumn{11}{|c|}{ Parents' LAHP (ref. $=$ not/somewhat important) } \\
\hline \multirow[t]{2}{*}{ Very important } & & & & & .86 & & .94 & & .83 & * \\
\hline & & & & & $(.07)$ & & $(.08)$ & & $(.08)$ & \\
\hline \multirow[t]{2}{*}{ Grade Point Average (GPA) } & & & & & & & 2.14 & $* * *$ & 2.14 & $* * *$ \\
\hline & & & & & & & (.13) & & $(.13)$ & \\
\hline \multicolumn{11}{|l|}{ Interactions } \\
\hline \multirow{2}{*}{ Latina/o * LAHP very important } & & & & & & & & & 1.72 & ** \\
\hline & & & & & & & & & $(.33)$ & \\
\hline \multirow[t]{2}{*}{ Constant } & 1.58 & $* * *$ & .75 & & .98 & & .13 & & .12 & $* * *$ \\
\hline & $(.06)$ & & $(.12)$ & & $(.19)$ & & $(.03)$ & & $(.03)$ & \\
\hline \multicolumn{11}{|c|}{ Outcome: Four-Year } \\
\hline & Model 1 & & Model 2 & & Model 3 & & Model 4 & & Model 5 & \\
\hline \multirow[t]{2}{*}{ Latina/o } & 0.36 & $* * *$ & .53 & $* * *$ & .67 & $* *$ & 1.00 & & .76 & \\
\hline & $(.03)$ & & $(.05)$ & & $(.09)$ & & $(.15)$ & & $(.14)$ & \\
\hline \multicolumn{11}{|l|}{ Parents' level of edu (ref. = less than HS) } \\
\hline \multirow[t]{2}{*}{ Some college or Associate's Degree } & & & 2.05 & $* * *$ & 1.51 & $* * *$ & 1.59 & $* * *$ & 1.59 & $* * *$ \\
\hline & & & $(.16)$ & $* * *$ & $(.14)$ & & $(.17)$ & & (.17) & \\
\hline \multirow[t]{2}{*}{ Four-year degree or higher } & & & 5.98 & & 4.15 & $* * *$ & 3.33 & $* * *$ & 3.29 & $* * *$ \\
\hline & & & $(.54)$ & & $(.45)$ & & $(.41)$ & & $(.40)$ & \\
\hline \multicolumn{11}{|c|}{ Immigrant generation (ref. = first generation) } \\
\hline Second generation & & & 1.13 & & .88 & & 1.06 & & 1.10 & \\
\hline & & & $(.20)$ & & $(.16)$ & & $(.22)$ & & $(.23)$ & \\
\hline Third + generation & & & .75 & & .53 & $* * *$ & .59 & $* *$ & .64 & $*$ \\
\hline & & & $(.12)$ & & $(.09)$ & & $(.12)$ & & $(.13)$ & \\
\hline Parents' LAHP (ref. $=$ not $/$ somewhat im & & & & & & & & & & \\
\hline Very important & & & & & .41 & $* * *$ & .52 & $* * *$ & .45 & $* * *$ \\
\hline & & & & & $(.04)$ & & $(.05)$ & & $(.05)$ & \\
\hline Grade Point Average (GPA) & & & & & & & 9.04 & $* * *$ & 9.03 & $* * *$ \\
\hline & & & & & & & $(.62)$ & & $(.62)$ & \\
\hline Interactions & & & & & & & & & & \\
\hline Latina/o * LAHP very important & & & & & & & & & 1.79 & $* *$ \\
\hline & & & & & & & & & $(.40)$ & \\
\hline Constant & 3.13 & $* * *$ & .43 & $* * *$ & 1.37 & & .01 & $* * *$ & .01 & $* * *$ \\
\hline & $(.10)$ & & $(.08)$ & & $(.32)$ & & $(.01)$ & & $(.01)$ & \\
\hline BIC & 11987 & & 11070 & & 10953 & & 9353 & & 9364 & \\
\hline
\end{tabular}

Model 2 includes added demographic controls; Model 4 includes school context measures

Coefficients for results are reported as Relative Risk Ratios (RRR). Robust standard errors reported in parentheses *** $\mathrm{p}<0.001, * * \mathrm{p}<0.01, * \mathrm{p}<0.05$

Table $2 \mathrm{~b}$, which shows selected coefficient from models predicting college attendance, is very similar to findings for college application. After controlling on demographic characteristics (see Model 2), Latinx students are no different from white students in their two-year college attendance rate. In addition, parents' strong LAHP is associated with a lower likelihood of attending both two-year and four-year college. When predicting four-year college attendance, Latinx 
students are less likely to attend compared to white students until Model 4, which includes students' GPA. We include the fit statistic called the Bayesian Information Criterion (BIC) in the model, which penalizes models for the inclusion of variables that do not significantly improve fit (Kass \& Raftery, 1995). The BIC compares the relative plausibility of two models, and a smaller BIC value is preferred. The slightly lower BIC value for Model 4 may suggest that the noninteractive model should be preferred. When viewing the coefficients and BIC statistic together, there is suggestive, but not conclusive, evidence of an interaction that suggests differential returns LAHP for white students.

Recall that one of our main research aims was to investigate whether Latinx parents who mimic the normative white preference of college residence - that of living away from home - received the same benefits from having that preference. Tables $2 \mathrm{a}$ and $2 \mathrm{~b}$ show some interesting effects regarding this research aim. For both application and attendance for twoyear and four-year college outcomes, the interaction between Latinx and LAHP of "very important" compared to "somewhat/not" important is statistically significant and positive. To better understand these interactions and provide substantive interpretations, we generated predicted probabilities by ethnoracial group at each level of parents' LAHP.

Predicted probabilities are shown in Figures $1 \mathrm{a}$ and $1 \mathrm{~b}$, and generally suggest that parents' LAHP has less of an impact on a Latinx student's likelihood to both apply and attend two- and four- year colleges compared to white students. The basic trend across predicted probabilities is that, among students whose parents have a strong LAHP, white and Latinx students are not much different from each other in their rates of applying to and attending college. Recall that previous research has consistently found that LAHP is negatively associated with any type of college attendance, but especially four-year college attendance. Our predicted probabilities suggest that both Latinx and white students whose parents' have a strong LAHP attend a two-year college at higher rates than Latinx and white students whose parents have a weaker LAHP. Both Latinx and white students whose parents' have a strong LAHP attend a four-year college at lower rates than Latinx and white students whose parent have a weaker LAHP (see Figure 1b). Predicted probabilities for application look similar to those of attendance (see Figure 1a). Importantly, there is little difference between Latinx and white students in their rates of application or attendance among those whose parents have a strong LAHP.

However, there is a substantial difference between Latinx students and white students among those whose parents have a weaker LAHP. Among white students, those whose parents have a weaker LAHP are more likely to both apply to and attend four-year colleges compared to white students whose parents have a strong LAHP. As shown in Figure 1a, white students whose parents encourage living away from home through a weaker LAHP are $16 \%$ more likely to apply to fouryear college (.69-.53) and 18\% more likely to attend four-year college (.55-.37) compared to white students whose parents have a stronger LAHP. Therefore, LAHP seems to matter a great deal for white students' likelihood of four-year collegegoing. For Latinx students, however, having parents with a weaker LAHP does not carry with it the same advantage. Those whose parents have a weaker LAHP are only $4 \%$ more likely to apply to four-year college (.54-.50) and $8 \%$ more likely to attend a four-year college (.41-.33).

Thus far, findings have suggested some interesting patterns concerning Latinx-white student differences in how parent's LAHP preference impacts the students' likelihood of applying to and attending college. While previous research suggests that parents' preference for their child to live away from home is advantageous for college going, our results suggest that this is simply not the case for Latinx students. In order to further understand how parents' LAHP may negatively or positively affect not only application and attendance patterns but also later stages in the college pipeline, we turn to students' attendance behaviors once they have enrolled in postsecondary schooling.

\section{Full-Time Attendance and Residence Patterns}

Our next two outcomes investigate two attendance patterns among high school graduates who have enrolled in a fouryear postsecondary institution: full-time versus part-time attendance and where students live while they are in college (see Tables 3 and 4, respectively). As shown in Table 3, findings suggest that Latinx students are less likely to be enrolled full-time among four-year college attendees. College students whose parents have a strong LAHP are also less likely to be enrolled full-time. The interactive model is not shown because analyses suggest that there are no ethnoracial group differences in the effect of LAHP on full-time enrollment at four-year universities. 
Table 3. Logistic regressions predicting enrollment status (full-time vs. part-time) during first year of college

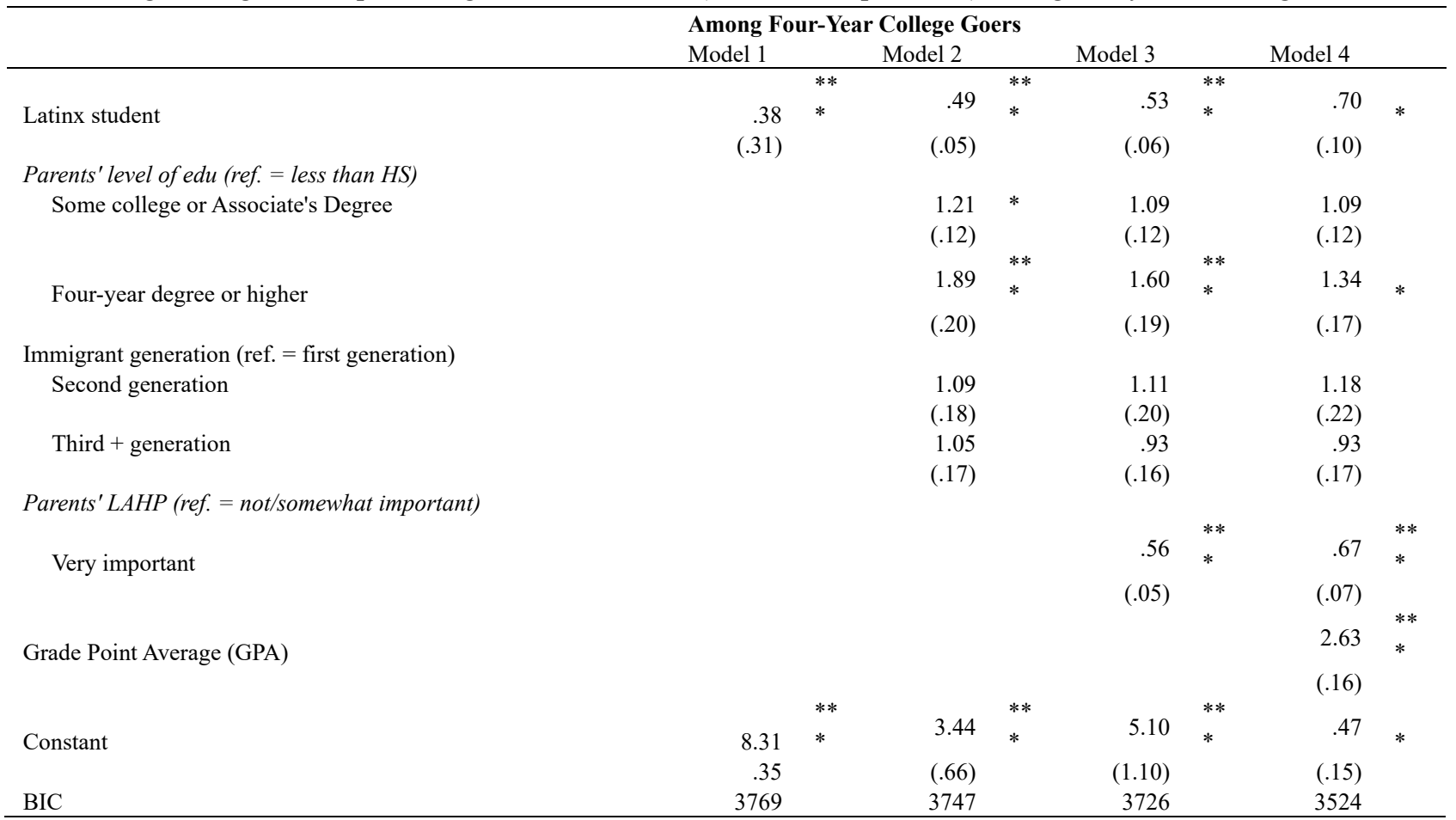

Coefficients for results are reported as odds ratios. Robust standard errors reported in parentheses $* * * \mathrm{p}<0.001, * * \mathrm{p}<0.01$, $* \mathrm{p}<0.05$ 
Table 4. Multinomial logistic regressions predicting residence during first year of college (reference $=$ live with parents)

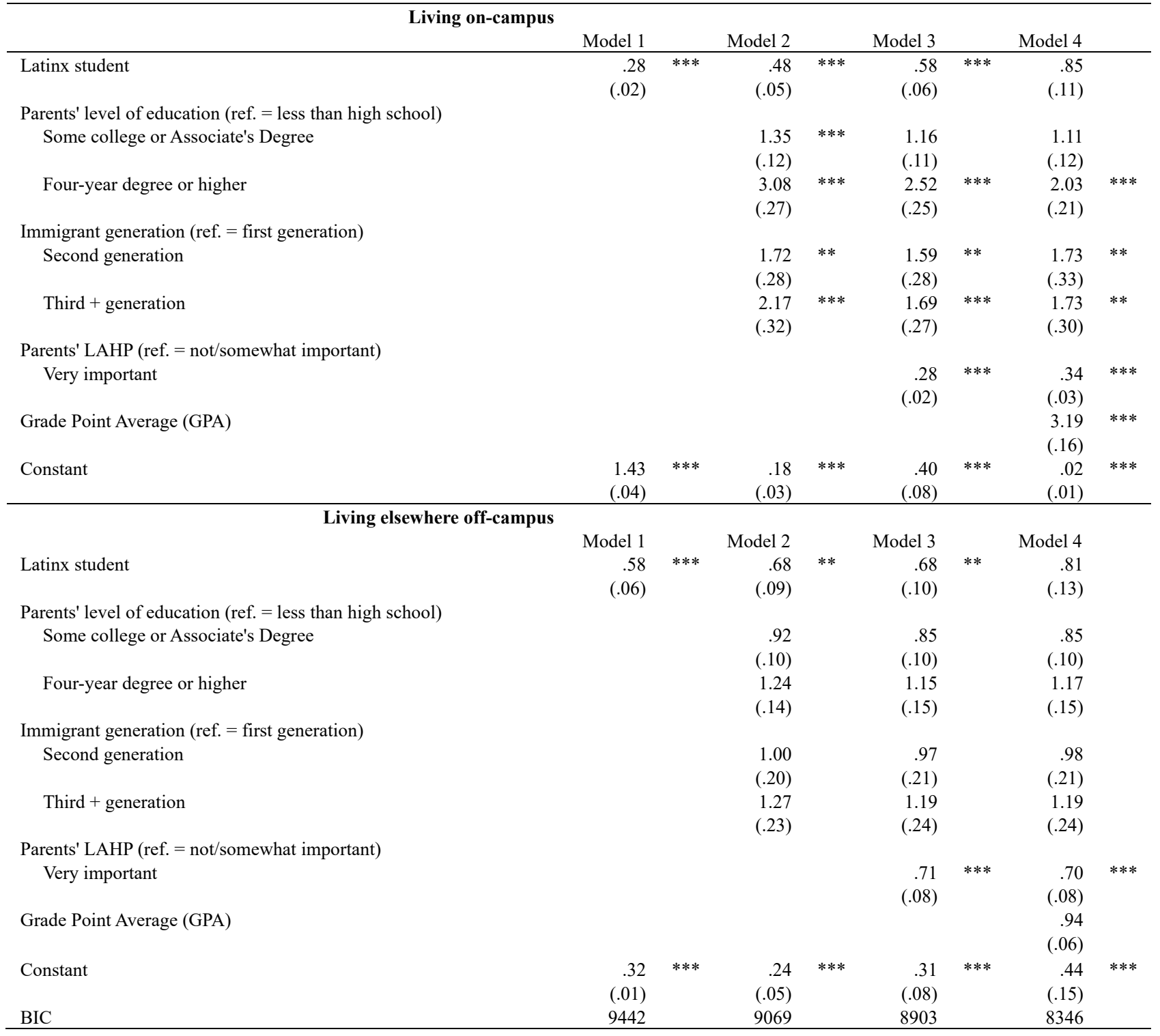

Coefficients for results are reported as Relative Risk Ratios (RRR). Robust standard errors reported in parentheses *** $\mathrm{p}<0.001, * * \mathrm{p}<0.01, * \mathrm{p}<0.05$ 


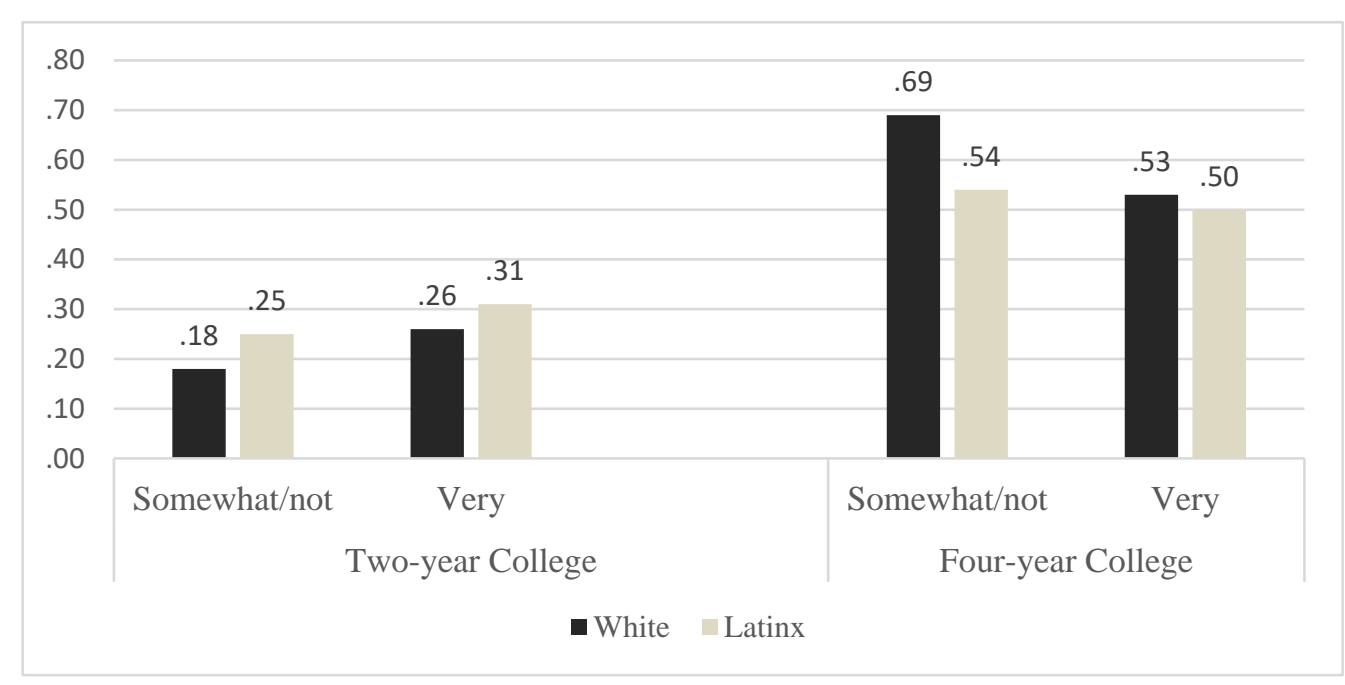

Figure 1a. Predicted Probabilities of Applying to Two-Year and Four-Year College, by Parents' LAHP and Ethnoracial Group Status

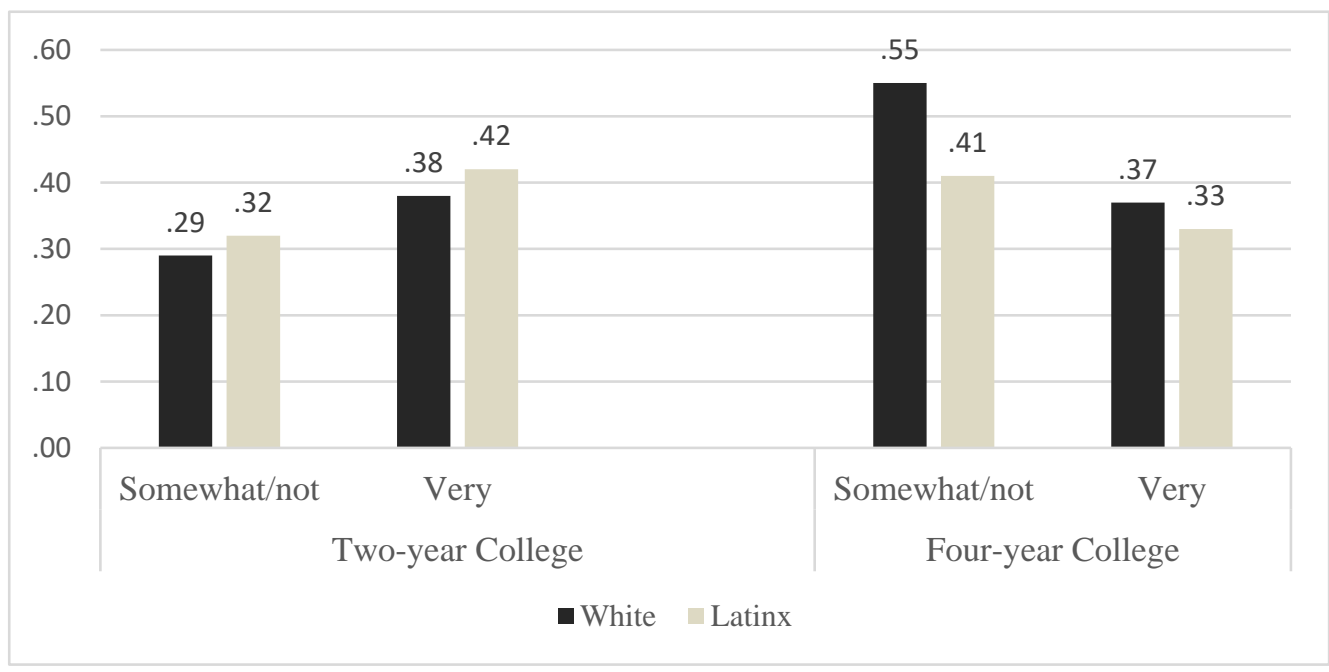

Figure 1b. Predicted Probabilities of Attending a Two-Year and Four-Year College, by Parents' LAHP and Ethnoracial Group Status

Concerning where students live during college, we are interested in whether or not parents' LAHP is associated with students' postsecondary residential living behavior. Table 4 shows models predicting whether students live on campus or in alternate off-campus housing compared to living with their parents (reference $=$ living with parents) with multinomial logistic regression. Findings suggest that Latinx students are less likely to live on-campus and elsewhere off-campus, even net of parents' preference that they live at home, until we control for students' GPA. Latinx students are no different from white students in their likelihood of living off-campus.

Although Table 4 suggests few differences by ethnoracial group in residential patterns, findings suggest that parents' preference for where their high school children live during college is associated with behavior. College students whose parents think it is "very" important for them to live at home during college are three times as likely to live at home during college (RRR = 3.01). Interestingly, parents' LAHP is also associated with a greater likelihood of college students' living off-campus.

\section{What Other Factors Might Affect Parents' LAHP?}

One of the challenging qualities of parents' LAHP is that while it does seem to predict postsecondary outcomes, it is unclear what having a preference that your adolescent lives at home actually means to parents. Supplemental descriptive and multivariate analyses were conducted in an effort to determine why parents might have a LAHP, whether or not the measures correlated with LAHP affect the associations between parents' LAHP and our college-going outcomes, as well 
as any differences between white and Latinx students in the effect of parents' LAHP. ${ }^{4}$ Descriptive analyses indicate that adolescents whose parents' have a strong LAHP take fewer actions of early college preparation activities (e.g., planning to take the SAT) and less often seek out information about colleges. In addition, parents who have a strong LAHP are less likely to have saved for college, but there are no indications that there are other socioeconomic differences correlated with parents' LAHP and economic hardship such as unemployment or reliance on adolescents for family income sources.

These supplemental descriptive analyses provide insight into what might be correlated with parents' LAHP. Importantly, descriptive analyses help us ascertain factors that are not correlated with parents' LAHP as well. For example, parents' LAHP is not correlated with adolescent or parent educational expectations, any of the questions related to explanations about why an adolescent did not go to college (e.g., cannot afford school, needs to support their family, does not like school, etc.), or parents' English language skills. Furthermore, multivariate analyses suggest that even when models control for college preparation activity measures and college savings efforts, results are similar to those presented in the current study. In other words, parents' LAHP is still associated with a variety of four-year college-going outcomes despite adding supplemental controls. In addition, adding these measures does not change the Latinx- white student differences in effects of parents' LAHP found when predicting college application (see Tables 2a and 2b), nor do the additional measures change the differences between Latinx and white students with our college-going outcomes.

Finally, we investigated whether geographic location changed the findings presented in the current study by excluding respondents from Texas and California. These two states have large community college systems (Snyder, de Brey, \& Dillow, 2016), which may influence students' postsecondary choice sets particularly around the idea of local access and living at home. Again, these models were consistent with findings presented in the current study.

\section{Discussion \& Conclusion}

Our findings suggest that while parents' weaker LAHP does increase a white student's likelihood of applying to and attending a four-year college, a Latinx student whose parents reflect a weaker LAHP does not accompany the same increase in likelihood of applying to and attending four-year college. As access to postsecondary institutions increases among all sociodemographic groups and students follow increasingly divergent pathways to the transition to college (Lee et al., 2018) and into adulthood (DeLuca, 2016), it is important to develop models that consider not only how students of color differ from white students in the transition to college, but also how statuses and behaviors that are favorable for whites are not likely to automatically translate into the same advantages for students of color.

Parents' LAHP is indicative of one way that college-going attitudes differ for Latinx and white students, and is an example of the way that postsecondary enrollment and attainment continues to be stratified through qualitative differences in decision-making. We argue that preference exemplifies how certain groups secure and maintain advantage in a stratified system (Lucas, 2001). Investigating differences between Latinx and white students illuminates differences in the patterns of college-going behaviors, where ideas and attitudes generally thought of as negatively associated with application and attendance may be positive for some groups or alternatively have no impact on behavior exhibited while enrolled.

Our study contributes knowledge to current research on students' pathways to postsecondary schooling. Within classic status attainment research (Bozick et al., 2010), we are interested in how families' sociodemographic origin creates intergenerational inequality or mobility. Although the main variables of interest (i.e. parent's level of education and parent's occupational status) continue to be used in research, our study takes this research tradition further by providing a more focused understanding of how family sociodemographic background can lead to inequality in the college pipeline. Rather than assuming that parents' LAHP has the same effect on all students, this study provides evidence that there are important differences between Latinx and white students in how parents' LAHP operates.

Similar to previous research on preferences to live at home (Desmond \& Lopez Turley, 2009; Lopez Turley et al., 2010), we found that parents' LAHP is associated with disadvantage for adolescents in the transition to college. Though preference is an important factor predicting outcomes in the postsecondary pipeline, effects of preference differed for Latinx students compared to white students. Descriptive analyses confirm that Latinx students report higher rates of parents' preference that they live at home being "very important." Preference does indeed seem to affect behavior along the postsecondary pipeline, and may potentially disadvantage students by sending them off on a path associated with greater difficulty in degree attainment. Why might parents' preference matter? Though some scholars have suggested parents' LAHP is akin to familismo, a cultural orientation that prioritizes family life over the individual (Desmond \& Lopez Turley, 2009), others argue that parents' LAHP is a function of socioeconomic constraint because living at home

${ }^{4}$ Separate models were also run by ethnoracial group, including interactions between parents' LAHP and parents' level of education. These analyses suggest no differences by gender, parents' level of education, income, or immigrant status for the effect of parents' LAHP on any of our outcome variables. 
during college saves money (Ovink \& Kalogrides, 2015). While both of these arguments have validity, they do not explain why parents' LAHP would be associated with poorer postsecondary outcomes; what is it about living at home that is detrimental to postsecondary attendance? The answer to this is likely complex, where some of the reasons lie with possible additional familial obligations (Espinoza, 2010) or lack of integration into college life in ways that limit social capital and academic engagement (Fischer, 2007).

Whatever the explanation for the association between parents' LAHP and postsecondary outcomes, it is important to emphasize our finding that, compared to whites, preference matters less to Latinx students for applying and attending four-year colleges and yet more for Latinx students attending two-year colleges. At the same time, Latinx parents who do not have a preference that their adolescent live at home during college are not gaining the same benefits as white parents with the same preference when predicting four-year college application or attendance. Therefore, even Latinx families who mimic white parent attitudes (and likely behavior) do not see the same returns as white families by espousing this attitude.

Future research should focus on expanding postsecondary models with the consideration that the same factors (e.g., parents' LAHP) do not function in the same way for all sociodemographic groups. Inequality for these groups is likely not only evident in disadvantages in outcomes along the postsecondary pipeline, but also concerning the ways that they interact with factors known to be beneficial to whites. Considering variation in the transition to postsecondary schooling may shift attention to factors that seem especially important for historically disadvantaged groups such as those from lower socioeconomic status backgrounds and students of color. For example, in the absence of parental knowledge in the transition to college, what might influence students' decisions? Though we do not include peer network information in our study due to data limitations, research has suggested that peers may be especially important for students whose parents did not attend college (Alvarado \& Turley, 2012; Perez \& McDonough, 2008). Incorporating a model that includes rich data on the social networks of adolescents may provide some insight concerning why parents' LAHP does not seem to matter as much for Latinx students in their transition to four-year college. In addition, future research on parental preferences would benefit from research that would continue to unearth what the parental preference to live at home while attending college actually means to parents and children as they are making decisions.

Despite limitations, our study expands upon what is known regarding the effects of parents' LAHP, and also provides insight into the ways that cultural norms about college-going affect stratification in the postsecondary pipeline. With the expansion of higher education and the increasingly diverse demographics, understanding quantitative and qualitative forms of inequality is necessary. Since parents' LAHPs may stem from accumulated forms of advantage and disadvantage, our research has policy implications that can began to remedy the gap. By understanding how parents' LAHP matters, high schools and postsecondary institutions together can provide information, resources and communicate with parents along the process to make sure that students have equal access to be able to make informed decisions about where to apply, attend, and approach their postsecondary institutions on matters that deal with residence and partaking in activities such as working part-time that come with living with parents. Postsecondary institutions in particular should work to better understand the reservations parents may have about sending their child away to college and find programmatic resources to alleviate parents' hesitations.

Our study also underscores the importance of parents' ideas and attitudes about college-going. Parents are crucial players in helping students make the transition to college and therefore a big source of inequality across ethnoracial groups is likely because of different levels of parental education. However, though Latinx parents may be less educated, this does not negate their importance in their child's life across this transition (Langenkamp \& Shifrer, 2018). Looking at parents as assets, despite lower levels of education, instead of a deficit, is an important needed change in perception when investigating different models of the transition to college. Research should continue to investigate differences in how parents react to the postsecondary arena as important points of inequality. Cultural knowledge about the transition to college is often hidden and patterned along ethnoracial lines. Our study highlights how not every factor predicting college outcomes works the same way for different ethnoracial groups, thereby contributing to our understanding of intergenerational inequality. Revealing ways that these hidden forms of knowledge produce very real inequality in outcomes is an important research agenda that involves communication with parents about cultural knowledge. At the same time, postsecondary institutions need to be accountable to adapting to the new postsecondary landscape of a more culturally and racially diverse student body and create opportunities for adolescents who do not live on campus. 


\section{References}

Acevedo, G. N. (2017). College-conocimiento: Toward an interdisciplinary college choice framework for Latinx students. Race, Ethnicity and Education, 20(6), 829-850. https://doi.org/10.1080/13613324.2017.1343294

Alexander, J. D., Cox, R. B., Jr., Behnke, A., \& Larzelere, R. E. (2017). Is All Parental "Noninvolvement" Equal? Barriers to Involvement and Their Relationship to Latino Academic Achievement. Hispanic Journal of Behavioral Sciences, 39(2), 169-179. https://doi.org/10.1177/0739986317700837

Alon, S. (2009). The evolution of class inequality in higher education competition, exclusion, and adaptation. American Sociological Review, 74(5), 731-755. https://doi.org/10.1177/000312240907400503

Alvarado, S. E., \& Turley, R. N. L. (2012). College-bound friends and college application choices: Heterogeneous effects for Latino and White students. Social Science Research, 41(6), 1451-1468. https://doi.org/10.1016/j.ssresearch.2012.05.017

Bean, F. D. (2015). Parent without papers the progress and pitfalls of Mexican-American integration. New York: Russell Sage Foundation.

Bourdieu, P., \& Passeron, J. C. (1977). Reproduction in Education, Society, and Culture. Sage Publications.

Bozick, R., Alexander, K., Entwisle, D., Dauber, S., \& Kerr, K. (2010). Framing the Future: Revisiting the Place of Educational Expectations in Status Attainment. Social Forces, 88(5), $2027-2052$. https://doi.org/10.1353/sof.2010.0033

Carbonaro, W., Ellison, B. J., \& Covay, E. (2011). Gender inequalities in the college pipeline. Social Science Research, 40(1), 120-135. https://doi.org/10.1016/j.ssresearch.2010.07.004

Ceja, M. (2004). Chicana college aspirations and the role of parents: Developing educational resiliency. Journal of Hispanic Higher Education, 3(4), 338-362. https://doi.org/10.1177/1538192704268428

Crisp, G., \& Nora, A. (2010). Hispanic student success: Factors influencing the persistence and transfer decisions of Latino community college students enrolled in developmental education. Research in Higher Education, 51(2), 175194. https://doi.org/10.1007/s11162-009-9151-X

Crosnoe, R. (2006). Mexican roots, American schools: Helping Mexican immigrant children succeed. Stanford University Press.

DeLuca, S. (2016). Coming of Age in the Other America. Russell Sage Foundation.

Desmond, M., \& Lopez Turley, R. N. (2009). The Role of Familism in Explaining the Hispanic-White College Application Gap. Social Problems, 56(2), 311-334. https://doi.org/10.1525/sp.2009.56.2.311

Espinoza, R. (2010). The Good Daughter Dilemma: Latinas Managing Family and School Demands. Journal of Hispanic Higher Education, 9(4), 317-330. https://doi.org/10.1177/1538192710380919

Fischer, M. J. (2007). Settling into campus life: Differences by race/ethnicity in college involvement and outcomes. The Journal of Higher Education, 78(2), 125-156. https://doi.org/10.1353/jhe.2007.0009

Grodsky, E. (2007). Compensatory Sponsorship in Higher Education. American Journal of Sociology, 112(Journal Article), 1662-1712. https://doi.org/10.1086/512707

Grodsky, E., \& Riegle-Crumb, C. (2010). Those Who Choose and Those Who Don't: Social Background and College Orientation. The ANNALS of the American Academy of Political and Social Science, 627(Journal Article), 14-35. https://doi.org/10.1177/0002716209348732

Johnson, D., Soldner, M., Leonard, J., Alvarez, P., Inkelas, K., Rowan Kenyon, H., \& Longerbeam, S. (2007). Examining Sense of Belonging Among First-Year Undergraduates From Different Racial/Ethnic Groups. Journal of College Student Development, 48(5), 525-542. https://doi.org/10.1353/csd.2007.0054

Klasik, D. (2012). The College Application Gauntlet: A Systematic Analysis of the Steps to Four-Year College Enrollment. Research in Higher Education, 53(5), 506-549. https://doi.org/10.1007/s11162-011-9242-3

Krogstad, J. M. (2016). 5 Facts about Latinos and Education. http://www.pewresearch.org/fact-tank/2016/07/28/5-factsabout-latinos-and-education/

Kurlaender, M. (2006). Choosing community college: Factors affecting Latino college choice. New Directions for Community Colleges, 2006(133), 7-16. https://doi.org/10.1002/cc.223

Langenkamp, A. G. (2017). Latino/a immigrant parents' educational aspirations for their children. Race Ethnicity and Education, 22(2), 231-249. https://doi.org/10.1080/13613324.2017.1365054 
Langenkamp, A. G., \& Hoyt, A. D. (2017a). The stratification of college-going Latinos' postsecondary destinations. Social Science Research, 67(Supplement C), 176-192. https://doi.org/10.1016/j.ssresearch.2017.05.005

Langenkamp, A. G., \& Hoyt, A. D. (2017b). Leaks in Latina/o Students' College-Going Pipeline: Consequences of Educational Expectation Attrition. Journal of Hispanic Higher Education, Journal Article, 1-15. https://doi.org/10.1177/15381927177498878

Langenkamp, A. G., \& Shifrer, D. (2018). Family Legacy or Family Pioneer? Social Class Differences in the Way Adolescents Construct College-Going. Journal of Adolescent Research, 33(1), 58-89. https://doi.org/10.1177/0743558416684951

Lee, T., Wickrama, K. A. S., O’Neal, C., \& Prado, G. (2018). Identifying diverse life transition patterns from adolescence to young adulthood: The influence of early socioeconomic context. Social Science Research, 70(Journal Article), 212-228. https://doi.org/10.1016/j.ssresearch.2017.12.001

Lopez, T. R. N., Desmond, M., \& Bruch, S. K. (2010). Unanticipated Educational Consequences of a Positive ParentChild Relationship. Journal of Marriage and Family, 72(5), 1377-1390. https://doi.org/10.1111/j.17413737.2010.00771.x

Lucas, S. R. (2001). Effectively Maintained Inequality: Education Transitions, Track Mobility, and Social Background Effects. American Journal of Sociology, 106(6), 1642-1690. https://doi.org/10.1086/321300

Massey, D. S., Charles, C. X., Lundy, G. F., \& Fischer, M. J. (2003). Source of the River: The Social Origins of Freshmen at America's Selective Colleges and Universities. Princeton University Press.

McDonough, P. M. (1997). Choosing Colleges: How Social Class and School Structure Opportunity. State University of New York Press.

Morgan, S. L. (2005). On the Edge of Commitment: Educational Attainment and Race in the United States. Stanford University Press.

Nuñez, A. M., McDonough, P. M., Ceja, M., \& Solorzano, D. (2008). Diversity within: Latino College Choice and Ethnic Comparisons. In C. A. Gallagher, C. Z. Charles, K. C. Torres, \& R. Brunn-Bevel (Eds.), Racism in Post-race America: New Theories, New Directions (Vol. 1-Book, Section, pp. 267-284). Social Forces Publishing.

Ovink, S. M., \& Kalogrides, D. (2015). No Place Like Home? Familism and Latino/a-White Differences in College Pathways. Social Science Research, 52(Journal Article), 219-235. https://doi.org/10.1016/j.ssresearch.2014.12.018

Ovink, S. M., \& Veazey, B. D. (2011). More than "Getting Us Through:" a case study in cultural capital enrichment of underrepresented minority undergraduates. Research in Higher Education, 52(4), 370-394. https://doi.org/10.1007/s11162-010-9198-8

Perez, P. A., \& Ceja, M. (2015). Higher Education Access and Choice for Latino Students: Critical Findings and Theoretical Perspectives (Vol. 12). Routlege. https://doi.org/10.4324/9781315718316

Perez, P. A., \& McDonough, P. M. (2008). Understanding Latina and Latino college choice: A social capital and chain migration analysis. Journal of Hispanic Higher Education, 7(3), 249-265. https://doi.org/10.1177/1538192708317620

Perna, L. W. (2000). Differences in the decision to attend college among African Americans, Hispanics, and Whites. Journal of Higher Education, 71(2), 117-141. https://doi.org/10.2307/2649245

Posselt, J. R., Jaquette, O., Bielby, R., \& Bastedo, M. N. (2012). Access Without Equity: Longitudinal Analyses of Institutional Stratification by Race and Ethnicity, 1972-2004. American Educational Research Journal, 49(6), 10741111. https://doi.org/10.3102/0002831212439456

Schudde, L. T. (2011). The causal effect of campus residency on college student retention. The Review of Higher Education, 34(4), 581-610. https://doi.org/10.1353/rhe.2011.0023

Shapiro, D., Dundar, A., Wakhungu, P. K., Yuan, X., Nathan, A., \& Hwang, Y. (2015). Completing college: A national view of student attainment rates-Fall 2009 cohort. National Student Clearinghouse Research Center.

Stanton-Salazar, R. D. (2001). Manufacturing hope and despair: The school and kin support networks of US-Mexican youth. Teachers College Press.

Taggart, A. (2018). Latina/o Students in K-12 Schools: A Synthesis of Empirical Research on Factors Influencing Academic Achievement. Hispanic Journal of Behavioral Sciences, 40(4), 448-471. https://doi.org/10.1177/0739986318793810

Turley, R. N. L., \& Wodtke, G. (2010). College residence and academic performance: Who benefits from living on campus? 
Urban Education, 45(4), 506-532. https://doi.org/10.1177/0042085910372351

Valencia, R. R. (2002). “Mexican Americans Don't Value Education!” On the Basis of the Myth, Mythmaking, and Debunking. Journal of Latinos and Education, 1(2), 81-103. https://doi.org/10.1207/S1532771XJLE0102_2

Yosso, T. J. (2006). Critical race counterstories along the Chicana/Chicano educational pipeline. Routledge. 
Appendix A. Description of Variables Used in Current Study

\begin{tabular}{|c|c|}
\hline Variable & Description \\
\hline \multicolumn{2}{|l|}{$\begin{array}{l}\text { Outcomes- taken from first postsecondary } \\
\text { institution attended }\end{array}$} \\
\hline Application to college & $\begin{array}{l}0=\text { no application, } 1=\text { two-year college including technical and } \\
\text { vocational programs, } 2=\text { four-year university }\end{array}$ \\
\hline Attend college & $\begin{array}{l}0=\text { no attendance, } 1=\text { two-year college including technical and } \\
\text { vocational programs, } 2=\text { four-year university }\end{array}$ \\
\hline $\begin{array}{l}\text { Attend full-time (among four-year college- } \\
\text { goers only) }\end{array}$ & $0=$ does not attend full time, $1=$ full-time attendance \\
\hline $\begin{array}{l}\text { Enrollment status (among four-year } \\
\text { college-goers only) }\end{array}$ & $\begin{array}{l}0=\text { living on campus, } 1=\text { living with parents, } 2=\text { living elsewhere off- } \\
\text { campus }\end{array}$ \\
\hline \multicolumn{2}{|l|}{ Independent Variables } \\
\hline Latinx & $1=$ Latin $x, 0=$ White, non-Latin $x$ \\
\hline Female & $1=$ Female, $0=$ Male \\
\hline Parent's level of education & $\begin{array}{l}\text { Higher of the two parents' education, measured as high school } \\
\text { graduate (reference), some college or associate's degree, four-year } \\
\text { degree or higher }\end{array}$ \\
\hline Immigrant generation $^{2}$ & $\begin{array}{l}\text { Measured as first generation (reference), second generation, } 1 \text { st or } \\
\text { 2nd Generation Immigrant, } 0=3 \mathrm{rd}+\text { Generation }\end{array}$ \\
\hline $\begin{array}{l}\text { Parents' income (ELS:2002 categorical } \\
\text { measure) }\end{array}$ & $\begin{array}{l}\text { less than } \$ 25,000 \text { (reference), } \$ 25,000-\$ 50,000, \$ 50,000-\$ 100,000 \text {, } \\
\text { and more than } \$ 100,000\end{array}$ \\
\hline Single parent household & $1=$ single parent household, $0=$ other family structure (reference) \\
\hline Parents' LAHP 3 & $0=$ not or somewhat important, $1=$ very important \\
\hline Cumulative Grade Point Average (GPA) & 12th grade GPA (transcript) \\
\hline $\begin{array}{l}\text { Percent of high school who are ethnoracial } \\
\text { minority students }\end{array}$ & $\begin{array}{l}\text { Percent minority students in the school in } 2001-2002 \text {, divided by } \\
10 \text { (Common Core of Data) }\end{array}$ \\
\hline High school urban location & $0=$ urban (reference), $1=$ suburban, $2=$ rural \\
\hline School sector & $0=$ public (reference), $1=$ Catholic, $2=$ other private \\
\hline
\end{tabular}

\footnotetext{
${ }^{1}$ We conducted supplemental analyses (not shown) that investigate whether LAHP affects two-year full-time college enrollment. It does not.

${ }^{2}$ Any students whose immigrant generation goes beyond grandparents are included in the third-generation category

${ }^{3}$ Parents' and students' preferences are generally correlated at .38, with no large differences between Latinx and white responses in parent/student agreement. Supplemental analyses restricted the sample to only parents and adolescents who agreed in their preference; these analyses suggest similar patterns of the way that preference affects our outcomes but with greater magnitude of effects. These analyses do not change any of the findings of preference as they relate to Latinx/white student differences.
}

\section{Copyrights}

Copyright for this article is retained by the author(s), with first publication rights granted to the journal.

This is an open-access article distributed under the terms and conditions of the Creative Commons Attribution license which permits unrestricted use, distribution, and reproduction in any medium, provided the original work is properly cited. 